\title{
Cannabis use in early psychosis is associated with reduced glutamate levels in the prefrontal cortex
}

\author{
Silvia Rigucci ${ }^{1,2,3} \cdot$ Lijing Xin $^{4}$ - Paul Klauser ${ }^{1,2}$ • Philipp S. Baumann ${ }^{1,2}$. \\ Luis Alameda ${ }^{1,2} \cdot$ Martine Cleusix $^{2} \cdot$ Raoul Jenni $^{2} \cdot$ Carina Ferrari $^{1}$ • \\ Maurizio Pompili ${ }^{3} \cdot$ Rolf Gruetter $^{5,6,7} \cdot$ Kim Q. Do $^{2} \cdot$ Philippe Conus $^{1}$
}

Received: 19 March 2017 /Revised: 6 August 2017 / Accepted: 7 September 2017 /Published online: 26 October 2017

(C) Springer-Verlag GmbH Germany 2017

\begin{abstract}
Rationale Recent studies have shown that cannabis may disrupt glutamate (Glu) signaling depressing Glu tone in frequent users. Current evidence have also consistently reported lower Glu-levels in various brain regions, particularly in the medial prefrontal cortex (mPFC) of chronic schizophrenia patients, while findings in early psychosis (EP) are not conclusive. Since cannabis may alter Glu synaptic plasticity and its use is a known risk factor for psychosis, studies focusing on Glu signaling in EP with or without a concomitant cannabis-usage seem crucial.
\end{abstract}

Silvia Rigucci and Lijing Xin are co first authors.

Kim Q. Do and Philippe Conus are equal contribution.

Kim Q. Do

Kim.Do@chuv.ch

1 TIPP (Treatment and Early Intervention in Psychosis Program); Service of General Psychiatry, Department of Psychiatry, Lausanne University Hospital (CHUV), Lausanne, Switzerland

2 Center for Psychiatric Neuroscience, Department of Psychiatry, Lausanne University Hospital (CHUV), Lausanne, Switzerland

3 Department of Neurosciences, Sensory Organs and Mental Health, Faculty of Medicine and Psychology, Sapienza University of Rome, Rome, Italy

4 Animal imaging and technology core (AIT), Center for Biomedical Imaging (CIBM), Ecole Polytechnique Fédérale de Lausanne, Lausanne, Switzerland

5 Laboratory of Functional and Metabolic Imaging, Ecole Polytechnique Fédérale de Lausanne, Lausanne, Switzerland

6 Departments of Radiology, University of Lausanne, Lausanne, Switzerland

7 Department of Radiology, University of Geneva, Geneva, Switzerland
Objective We investigate the effect of cannabis use on prefrontal Glu-levels in EP users vs. both EP non-users and healthy controls (HC).

Methods Magnetic resonance spectroscopy was used to measure $\left[\mathrm{Glu}_{\mathrm{mPFC}}\right]$ of $35 \mathrm{EP}$ subjects (18 of whom were cannabis users) and $33 \mathrm{HC}$. For correlative analysis, neuropsychological performances were scored by the MATRICS-consensus cognitive battery.

Results $\left[\mathrm{Glu}_{\mathrm{mPFC}}\right]$ was lower in EP users comparing to both $\mathrm{HC}$ and EP non-users ( $p<0.001$ and $p=0.01$, respectively), while no differences were observed between EP non-users and HC. A greater $\left[\mathrm{Glu}_{\mathrm{mPFC}}\right]$-decline with age was observed in EP users $(r=-.46 ; p=0.04)$, but not in EP non-users or HC. Among neuropsychological outcomes, working memory was the only domain that differentiates patients depending on their cannabis use, with users having poorer performances.

Conclusions Cannabis use is associated with reduced prefrontal $\left[\mathrm{Glu}_{\mathrm{mPFC}}\right]$ and with a stronger Glu-levels decline with age. Glutamatergic abnormalities might influence the cognitive impairment observed in users and have some relevance for the progression of the disease.

Keywords Magnetic resonance spectroscopy - Glutamate · Cannabis $\cdot$ Early psychosis $\cdot$ Cognition

\section{Introduction}

$\Delta$ 9-tetrahydrocannabinol (THC), the primary psychoactive cannabinoid present within cannabis, exerts its effect by binding the cannabinoid receptors type 1 (CB1R) that are widely distributed in the brain. Consequently, the long-term use of cannabis might cause alterations in both morphology and function of brain regions rich in CB1R (i.e., prefrontal cortex, hippocampus, amygdala, cerebellum), but so far findings are 
still controversial (Lorenzetti et al. 2015). If the impact of cannabis use on brain structure is largely investigated, still little is known about the effect of cannabis on brain tissue's neurochemical profiles. Proton magnetic resonance spectroscopy $\left({ }^{1} \mathrm{H}\right.$ MRS $)$, is a non-invasive measurement technique that enables the in vivo detection of a wide range of neurometabolites including neurotransmitters. Among them, glutamate (Glu) could be considered as a promising candidate to capture the effects of cannabis on the brain (for a review see Colizzi et al. 2016). There is indeed a large body of evidence accumulated from animal studies demonstrating that in vivo administration of THC affects the release of Glu in the prefrontal cortex (PFC), and that this process is mediated by CB1R (Pistis et al. 2001). Specifically, the administration of THC depresses endocannabinoid-mediated glutamatergic synaptic transmission, affecting the release, the enzyme activity, and the expression of both Glu-receptors and transporters (Colizzi et al. 2016). Also, prolonged THC exposure affects Glu synaptic plasticity by a functional tolerance mechanism, reducing the ability of CB1R-agonists to inhibit Glu synaptic transmission (Mato et al. 2005).

The application of MRS to investigate the effect of cannabis in humans, although limited by the paucity of studies, tends to replicate findings of animal models. To the best of our knowledge, only four authors examined in vivo Glurelated metabolites levels in cannabis users, while only one prior study focused on a population of schizophrenia patients with a concomitant cannabis usage. Lower levels of Glu, Nacetylaspartate (NAA), and myo-inositol (mIns) were observed in regular cannabis users without a psychiatric disorder compared to control subjects, in regions known to be associated with substance use: including the dorsal striatum in 27 subjects (Muetzel et al. 2013), the anterior cingulate cortices in 17 and 13 cannabis users, respectively (Prescot et al. 2011, 2013), and the basal ganglia and thalamus in 24 individuals without psychosis (Chang et al. 2006). The only study focusing on patients with schizophrenia found a specific effect of substance use on reducing levels of Glu plus Glutamine (Gln) (Bernier et al. 2016).

The impact of THC on Glu-signaling is of considerable interest as it offers a possible key to unravel the neurobiological substrate of the clinical phenotype of cannabis-associated psychosis. Supporting an integrated hypothesis, several lines of evidences point indeed to there being an hypo-functioning Glu neurotransmission leading to the presynaptic dopamine dysfunction consistently reported in schizophrenia, which may provide the best explanation of all the clinical aspects of this heterogeneous and complex disorder (Howes et al. 2015). Furthermore, recent studies have shown decreased Glu levels in schizophrenia patients when compared to healthy individuals, particularly in medial frontal region (Marsman et al. 2013). Moreover, chronicity in schizophrenia was found to be related to decreased prefrontal Glu (Natsubori et al. 2014), Glx (Glu + Gln) and NAA levels (Liemburg et al. 2016); with evidence for a time-depending role for Glu (Merritt et al. 2016), whereas findings in early psychosis (EP) are still controversial (for a review see Treen et al. 2016). While the studies presented above provide evidence that THC leads to glutamatergic dysfunction, they do not unequivocally identify the specific brain circuits or regions involved. The PFC has been suggested, among others, as a potential target-site as glutamatergic projections to the midbrain regulate midbrain dopamine neurons (Howes et al. 2015). Furthermore, the prefrontal cortical and subcortical changes in metabolism occurring within cannabis users are thought to disturb prefrontal cortical maturation, which could ultimately give rise to neurobiological impairments (Zamberletti et al. 2014; Rubino et al. 2015).

THC seems therefore to have the potential to disrupt the normal interplay between neurotransmitters in PFC, particularly Glu, and may bear relevance in understanding THCassociated cognitive and clinical dimensions. The aim of the present study was to employ ${ }^{1} \mathrm{H}$ MRS for investigating Glu levels in the mPFC of EP cannabis users compared with their non-using counterpart and normative healthy controls.

Based on this background we hypothesized:

(i) a reduced glutamate level in EP subjects who used cannabis compared to EP non-users and healthy controls, respectively

(ii) the absence of a difference between EP non-users and $\mathrm{HC}$ in terms of [ $\left.\mathrm{Glu}_{\mathrm{mPFC}}\right]$

(iii) a correlation between level of cognitive deficits in EP users and pre-frontal glutamatergic dysfunction

\section{Method}

\section{Subjects}

Thirty-five EP subjects, of whom 18 were currently cannabis users, were recruited from the Treatment and Early Intervention in Psychosis Program (TIPP), of the University Hospital in Lausanne (Baumann et al. 2013). The TIPP is a specialized 3-year early psychosis programme. Entry criteria to the program are (i) age between 18 and 35 years; (ii) residence in the catchment area (Lausanne and surrounding); (iii) meeting threshold criteria for psychosis, as defined by the 'Psychosis threshold' subscale of the Comprehensive Assessment of At Risk Mental States (CAARMS) (Yung et al. 2005); and (iv) not more than 6 months of treatment with antipsychotic medication for the psychotic disorder. The CAARMS is a clinical tool to assess if subjects meet criteria for being at ultra-high risk for onset of first psychotic disorder. The "Psychosis threshold" subscale allows assessing if a 
subject fulfills criteria for a "full blown" psychotic episode. The diagnostic assessment for TIPP patients is the result of an expert consensus and is based on the following elements: (Andreasen et al. 2010) diagnosis reported by treating psychiatrist in all medical documents and at the end of any hospitalization; (Arseneault et al. 2002) longitudinal assessment by clinical case managers over the 3 years of treatment. The consensus diagnosis procedure realized by a senior psychiatrist and the senior psychologist who is in charge of scale-based assessment over the treatment period. They both review the entire file once after 18 months and again after 36 months or at the end of treatment and conduct a diagnostic process based on DSM-IV criteria (APA 1994) discussing any unclear issue with the clinical case manager. In this paper, only the final diagnosis, defined at the end of TIPP treatment period, is considered. Thirty-three healthy controls matched on gender, age, and handedness were recruited from similar geographic and socio-demographic areas and assessed by the Diagnostic Interview for Genetic Studies (Preisig et al. 1999). Major mood, psychotic, or substance-use disorder and having a first-degree relative with a psychotic disorder were exclusion criteria for controls. Neurological disorders and severe head trauma were exclusion criteria for all subjects. 31 of the 35 patients were on antipsychotic medication at the time of this study, with an average medication of $329.3 \pm 212.1 \mathrm{mg}$ chlorpromazine equivalent dose (CPZ) (Andreasen et al. 2010) (Table 1). Informed written consent in accordance with our institutional guidelines (protocol approved by the Ethic Committee of Lausanne University) was obtained for all the subjects.

\section{Assessment of cannabis use}

A detailed history of illicit drug use (cannabis, stimulants and any other recreational drug) was taken using a specially designed questionnaire (the TIPP Initial Assessment Tool: TIAT). This allows a detailed assessment of lifetime patterns of cannabis use, including frequency and duration of use and age at first use. The measures of exposure to cannabis use included in the analyses were (a) current cannabis use (is the subject currently using cannabis: no $=0$; yes $=1$ ); (b) lifetime frequency of cannabis use [the frequency that characterized the subject's most consistent pattern of use: at weekends or less frequently $($ occasional $)=0$; every day $($ daily $)=1]$; and (c) age at first use (the age when the subject started to use cannabis regularly: prior to age 15 years $=0$; above age 15 years $=1$ ). This cut-off was based on previous studies showing an increased risk of mental health disorders in users of cannabis before the age of 15 (Arseneault et al. 2002; Fontes et al. 2011). On the basis of this data, EP patients were dichotomised between (a) regular cannabis users and (b) occasional users.

\section{Neuropsychological assessment}

Neuropsychological assessment was made according to the "Measurement and Treatment Research to improve Cognition in Schizophrenia" (MATRICS Consensus Cognitive Battery), excluding the Mayer-Salovey-Caruso Emotional Intelligence Test (MSCEIT), which does not "translate" well into French as an index of social cognition. This includes six factors (speed of processing, sustained attention/vigilance, working memory, verbal and visual learning, reasoning and problem-solving) (Nuechterlein et al. 2008), on the basis of the following subtests: Trail Making Test (TMT): part A; Symbol Coding - Brief Assessment of Cognition in Schizophrenia (BACS): symbol coding; Verbal (category) Fluency: animal naming; Continuous Performance TestIdentical Pairs (CPT-IP); Spatial Span-Wechsler Memory Scale-Third Edition (WMS-III): spatial span; Scale-Third Edition; Letter-Number Span (LNS); Hopkins Verbal Learning Test-Revised (HVLT-R); Brief Visuospatial Memory Test-Revised (BVMT-R); Mazes - Neuropsychological Assessment Battery (NAB): mazes. Median time from admission to the TIPP programme and to MRS scan was $375.03 \pm 331.3$ days. Median time from MRS scan to clinical assessment was $20.6 \pm 6$ days.

Table 1 Subjects characteristics

\begin{tabular}{|c|c|c|c|c|}
\hline & $\begin{array}{l}\text { EP users }(\mathrm{N}=18) \\
\text { Mean } \pm \mathrm{SD}\end{array}$ & $\begin{array}{l}\text { EP non-users }(\mathrm{N}=17) \\
\text { Mean } \pm \mathrm{SD}\end{array}$ & $\begin{array}{l}\mathrm{HC}(\mathrm{N}=33) \\
\text { Mean } \pm \mathrm{SD}\end{array}$ & Statistics \\
\hline Age, years & $27.1(6.2)$ & $24.8(6.1)$ & $25.3(4.5)$ & $F(2.67)=1.1 p=0.3$ \\
\hline Gender, $\mathrm{M} / \mathrm{F}$ & $13 / 5$ & $9 / 8$ & $16 / 17$ & $\chi^{2}=4.1 p=0.1$ \\
\hline Handedness R/L/A & $15 / 2 / 1$ & $14 / 2 / 1$ & $26 / 7 / 0$ & $\chi^{2}=5 p=0.7$ \\
\hline Duration of illness, days & $774.8(772.9)$ & $867.3(754.4)$ & - & $\mathrm{t}=-.38 p=0.7$ \\
\hline Medication dose, $\mathrm{mg}^{\mathrm{a}}$ & $320.9(336.2)$ & $331.3(227.1)$ & - & $\mathrm{t}=-.1 p=0.9$ \\
\hline Patterns of use (every day users, \%) & $8(44.4)$ & - & - & \\
\hline
\end{tabular}

$E P$ early psychosis, $H C$ healthy controls

${ }^{\mathrm{a}}$ Four patients were not on antipsychotics at time of scanning 


\section{${ }^{1} \mathrm{H}$ magnetic resonance spectroscopy}

${ }^{1} \mathrm{H}$ MRS measurements were performed on the 3T MR scanner (Magnetom TimTrio, Siemens Healthcare, Erlangen, Germany) with a transverse electromagnetic (TEM 3000) head coil (MR Instruments, Inc). The magnetic field homogeneity was optimized by adjusting first- and second-order shims using FAST(EST)MAP (Gruetter 1993; Gruetter and Tkac 2000).

In vivo $1 \mathrm{H}$ MR spectra were acquired from the medial prefrontal cortex (mPFC; Fig. 1a) using a short-TE spin-echo full-intensity acquired localized single voxel spectroscopy technique (SPECIAL) (Mekle et al. 2009; Mlynarik et al. 2006). Outer volume suppression (OVS) (Tkac et al. 2001) and water suppression with variable-pulse power and optimized relaxation delays (VAPOR) (Tkac et al. 1999) were applied prior to the SPECIAL localization sequence. The following scan parameters were used: volume of interest $(\mathrm{VOI}=20 \times 20 \times 25 \mathrm{~mm} 3)$, echo time/repetition time $(\mathrm{TE} /$ $\mathrm{TR})=6 / 4000 \mathrm{~ms}$, acquisition bandwidth $=2 \mathrm{kHz}$, number of averages $=148$, vector size $=2$.

\section{Spectral quantification}

To obtain $\left[\mathrm{Glu}_{\mathrm{mPFC}}\right]$ water suppressed in vivo, ${ }^{1} \mathrm{H} \mathrm{MR}$ spectra were analyzed by LCModel (Stephen Provencher, Inc) using a basis-set consisting of 20 simulated individual metabolite spectra: alanine (Ala),

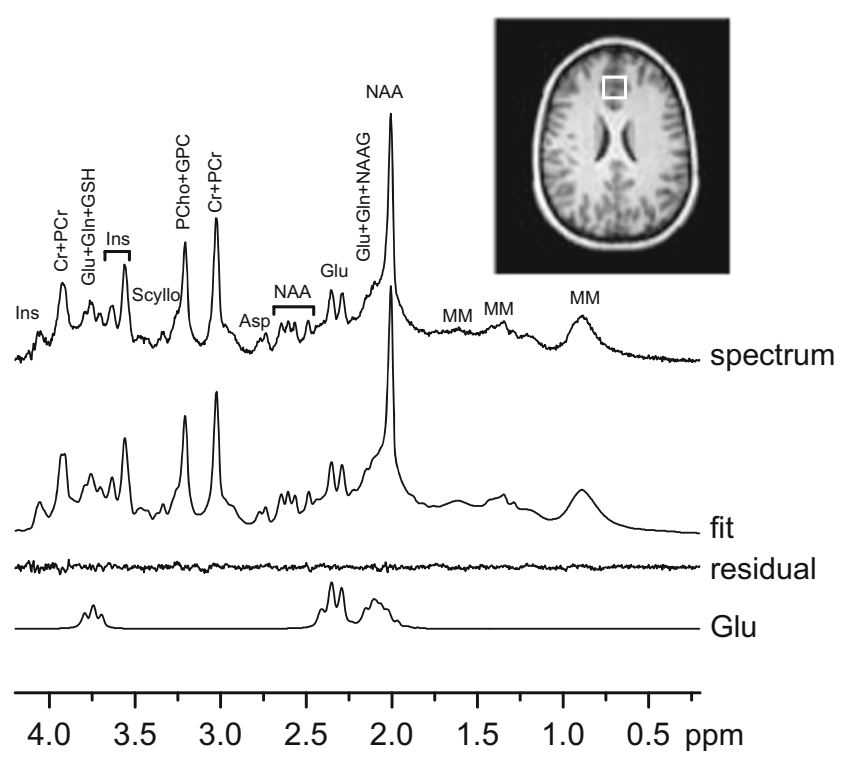

Fig. 1 A ${ }^{1} \mathrm{H}$ MR spectrum of medial prefrontal cortex $\left(\right.$ VOI $=20 \times 20 \times 25 \mathrm{~mm}^{3}$ ) shown in the transverse slice of T1weighted MPRAGE images) acquired with the SPECIAL sequence at $3 \mathrm{~T}(\mathrm{TE} / \mathrm{TR}=6 / 4000 \mathrm{~ms}$, number of averages $=148)$, the corresponding LCModel spectral fit, fit residual and Glu fit aspartate (Asp), phosphocreatine $(\mathrm{PCr})$, creatine $(\mathrm{Cr}), \gamma$ aminobutyric acid (GABA), glutamine (Gln), glutamate (Glu), phosphorylcholine ( PCho), glycerophosphorylcholine (GPC), gluthathione (GSH), glucose (Glc), lactate (Lac), glycine (Gly), myo-inositol (mIns), N-acetylaspartylglutamate (NAA), Nacetylaspartylglutamate (NAAG), ascorbate (Asc), phosphoryl-ethanolamine (PE), scylloinositol (Scyllo), taurine (Tau), and an experimentally measured macromolecule baseline. Unsuppressed water $1 \mathrm{H}$ MR spectra were used as an internal reference for the absolute quantification of metabolite concentrations. Tissue composition inside the MRS volume used for water content calculation and partial volume correction was calculated based on the segmentation of magnetization-prepared rapid gradientecho (MPRAGE) (Mugler and Brookeman 1990) images $(\mathrm{TE} / \mathrm{TR}=2.98 / 2300 \mathrm{~ms}, \mathrm{TI}=900 \mathrm{~ms}$, flip angle $=9$ degree, $\mathrm{FOV}=240 \times 256 \mathrm{~mm} 2$, matrix $=240 \times 256$, slice thickness $=1.2 \mathrm{~mm}$ ) MPRAGE images using an in-house software (more details are reported elsewhere, Xin et al. 2016).

\section{Statistical analysis}

Statistical analyses were performed with SPSS (SPSS, Chicago, Illinois). Differences between groups in sociodemographics and clinical characteristics were assessed with Chi-square or $T$ test as appropriate. To investigate the effect of cannabis on prefrontal Glu levels, an univariate analyses of covariance (ANCOVAs) was used, with mean [ $\left.\mathrm{Glu}_{\mathrm{mPFC}}\right]$ entered as dependent variable, group as fixed factor (EP-users; EP non-users and $\mathrm{HC}$ ); age and gender as covariates of no interest. To examine the effect of cannabis across the users' sample, the same analyses were performed with frequency of use (never or occasional vs. daily), and age at first use (< or $>15$ years) separately entered as fixed factors. The same analyses were repeated entering alcohol use, number of cigarettes smoked and medication (CPZ equivalents) as nuisance variables. Analyses performed for Gln, NAA and GABA are shown in Supplementary materials.

Differences in neuropsychological measures between EPusers, non-users, and controls were investigated using a multivariate analysis of covariance (MANCOVA).

Finally, correlations between mean $\mathrm{Glu}_{\mathrm{mPFC}}$ values and cognitive measures were tested with Pearson's correlation coefficient. The correlation analyses were performed only on neuropsychological domain that differentiate within patients depending on their cannabis usage. A Fisher r-to-z transformation of the correlation coefficients was used to compare the strength of the correlations between groups. (Calculation for the test of the difference between 2 independent correlation coefficients is available from http://quantpsy.org). 


\section{Results}

\section{Socio-demographic characteristics}

There were no significant differences in age, gender, and handedness between $\mathrm{EP}$ subjects and $\mathrm{HC}$, indicating that patients and controls were well matched for these factors. As expected, patients had a lower level of education than controls $(t=4.88, p=0.001)$. PANSS was similar for the two groups, with EP users having 62.7(17.7) and EP nonusers 64.1(15.8) mean total PANSS score $[\mathrm{t}=-.26$ $p=0.7]$. At 36 months follow-up within the users, 11 patients were diagnosed with schizophrenia, 4 with brief psychotic disorder, 3 with schizoaffective disorder. Among the non-users group, 9 patients were diagnosed with schizophrenia, 4 with schizoaffective disorder; 2 with bipolar disorder and another 2 with brief psychotic disorder. Main clinical characteristics are presented in Table 1.

\section{Cannabis and patterns of use}

$51.4 \%(N=18)$ of patients were currently cannabis users, 10 among them were occasional users (used weekly or less), and another 8 were daily users (daily usage or more than 3 times per week). The mean age at onset of cannabis use were $17 \pm 4.5$ years, with cannabis users being more likely alcohol users $[\chi 2(4,5), p=0.02]$ and cigarettes smokers $[(\chi 2(18,2), p<0.001])$ compared to non-users. There were no statistical differences in age, gender, medication ( $\mathrm{Cpz}$ equivalents) and duration of illness between the two groups (users Vs non-users). EP users had a lower level of education than non-users $(t=3.82, p=0.001)$. Other drugs (cocaine, stimulants, heroine, and opioids) were minimally used, with $1 \mathrm{EP}$ users having a concomitant, sporadic use of cocaine. Within the EP non-users group there was only 1 subject who had used cannabis in the past, occasionally and during 3 years, but who had stopped smoking two years before entering the study.

\section{Neuropsychological performance}

Complete neuropsychological performances are detailed in Table 2. As expected, neuropsychological performances were deeply affected in patients as compared to healthy controls particularly when considering EP users, while a less cognitive impairment was observed in EP non-users $F(2.57)=; p<0.001 ; d=0.51$. Working memory was the only domain found to be impaired in EP users compared to their non-using counterpart, and that entered the subsequent correlative analyses. Patterns of cannabis use were not significantly related to any cognitive measures; age at which regular cannabis use commenced did not have a significant effect either.

\section{$\left[G_{\text {LPFC }}\right]$ and cannabis use}

$\left[\mathrm{Glu}_{\mathrm{mPFC}}\right]$ was measured by short-TE ${ }^{1} \mathrm{H}$ MRS at $3 \mathrm{~T}$ and quantified using LCModel with CRLBs of $2.0 \pm 0.5 \%$ (mean \pm SDMean). [ $\left.\mathrm{Glu}_{\mathrm{mPFC}}\right]$ levels in EP users were significantly lower $(12.7 \pm 1.3 \mu \mathrm{mol} / \mathrm{g})$ than in both EP non-users $(13.9 \pm 1.5 \mu \mathrm{mol} / \mathrm{g})$ and $\mathrm{HC}(14.2 \pm 0.9 \mu \mathrm{mol} / \mathrm{g})$, $[\mathrm{F}(4.66)=7.1 ; p=0.002]$ with a medium effect size of $d=0.4$. Bonferroni post-hoc tests showed a significant reduction on $\left[\mathrm{Glu}_{\mathrm{mPFC}}\right]$ levels between EP-users and healthy controls $(p=0.001)$ and also between EP users and EP non-users $(p=0.013)$; while controls and EP non-users did not differ $(p=0.9)$.

When alcohol and cigarette intake, as well as medication (CPz equivalents) was set as nuisance factors in the model, all results retain their statistical significance.

Patterns of use did not significantly affect [ $\left.\mathrm{Glu}_{\mathrm{mPFC}}\right]$ levels $[\mathrm{F}(4,16)=0,1 ; p=0.7]$, albeit every day users having a slightly lower mean $\left[\mathrm{Glu}_{\mathrm{mPFC}}\right]$ levels $(12.5 \pm 1.3 \mu \mathrm{mol} / \mathrm{g})$ than occasional users $(13.2 \pm 0.9 \mu \mathrm{mol} / \mathrm{g})$, respectively. Finally, we evaluated the effect of age at first use, and found no significant differences in mean $\left[\mathrm{Glu}_{\mathrm{mPFC}}\right]$ levels between early ( $<15$ years) and late onset cannabis users ( $>15$ years) (Fig. 2$)$.

\section{Correlation between $\left[G L U_{m P F C}\right]$ and clinical characteristics}

$\left[\mathrm{GLU}_{\mathrm{mPFC}}\right]$ levels were not related to mean duration of illness $(r=-11, p=0.3)$, and neither to PANSS total scores $(r=-0.4 ; p=0.4)$. CPZ equivalents displayed a significant positive association with $\left[\mathrm{GLU}_{\mathrm{mPFC}}\right](r=.34 ; p=0.03)$.

\section{Correlation between [GLU $U_{\mathrm{mPFC}}$ ] and working memory}

In the sample as a whole (both EP and controls), mean $\left[\mathrm{GLU}_{\mathrm{mPFC}}\right]$ levels positively correlate with working memory (WM) $(r=.37, p=0.002)$. When running correlations separately for each group, this correlation still remains significant in controls $(r=.43 ; p=0.01)$ but not in EP non-users or EP users. After controlling for age and years of education, results maintain significance. EP users and controls demonstrate, at trend level, differences in correlation coefficients of $\left[\mathrm{GLU}_{\mathrm{mPFC}}\right]$ with $\mathrm{WM}(\mathrm{z}=1.4$, $p=0.07$ ) (Fig. 3).

\section{Correlations between mean $\mathrm{Glu}_{\mathrm{mPFC}}$ and age}

Age was significantly negatively associated with mean $\left[\mathrm{Glu}_{\mathrm{mPFC}}\right]$ levels in the combined sample $(r=-.30$; $p=0.006)$ and independently in EP $(r=-.36 ; p=0.02)$. 
Table 2 Neuropsychological performance in EP cannabis users, non-users, and healthy controls

\begin{tabular}{|c|c|c|c|c|c|c|c|}
\hline \multirow{2}{*}{$\begin{array}{l}\text { Cognitive domain } \\
\text { - subtests }\end{array}$} & \multicolumn{4}{|l|}{ Mean (SD) } & \multicolumn{3}{|l|}{$P^{\mathrm{a}}$ value } \\
\hline & $\begin{array}{l}\text { EP users }{ }^{b} \\
(N=18)\end{array}$ & $\begin{array}{l}\text { EP non-users }{ }^{\mathrm{c}} \\
(N=17)\end{array}$ & $\mathrm{HC}^{\mathrm{d}}(N=33)$ & $\begin{array}{l}\text { Effect size } \\
\text { (Cohen's } d \text { ) }\end{array}$ & $\begin{array}{l}\text { EP users Vs EP } \\
\text { non-users }\end{array}$ & $\begin{array}{l}\text { EP users } \\
\text { Vs HC }\end{array}$ & $\begin{array}{l}\text { EP non-users } \\
\text { Vs HC }\end{array}$ \\
\hline Speed of processing & $34.3(14.7)$ & $44.2(11.7)$ & $54.3(5.8)$ & 0.42 & .05 & .001 & .02 \\
\hline - TM- A & $35.9(14)$ & $45(9.8)$ & $50(13.2)$ & 0.21 & .20 & .002 & .79 \\
\hline $\begin{array}{c}\text { - Symbol coding } \\
\text { (BACS) }\end{array}$ & $33.1(13.3)$ & $42.7(10.6)$ & $45.8(14.2)$ & 0.16 & .18 & .01 & .92 \\
\hline - Verbal fluency & $44.4(13.4)$ & $49.1(13.5)$ & $56.8(11.4)$ & 0.17 & .98 & .006 & .23 \\
\hline \multicolumn{8}{|l|}{ Sustained attention } \\
\hline • CPT & $37.9(11.7)$ & $41.4(11.7)$ & $52.5(8.5)$ & 0.31 & .24 & .001 & .007 \\
\hline Working Memory & $41.2(12.7)$ & $49.2(6.6)$ & $56.8(7.5)$ & 0.36 & .07 & .001 & .06 \\
\hline - LNS & $41(11.4)$ & $50.2(8.1)$ & $54.5(6.5)$ & 0.33 & .02 & .001 & .41 \\
\hline - Spatial span (WMS) & $44.4(12.1)$ & $48.5(6.9)$ & $57.3(9.2)$ & 0.27 & .82 & .001 & .03 \\
\hline \multicolumn{8}{|l|}{ Problem Solving } \\
\hline$\cdot$ Mazes-NAB $^{\dagger}$ & $43(12.6)$ & $40.9(11.5)$ & $56.6(7.9)$ & 0.34 & .85 & .002 & .001 \\
\hline \multicolumn{8}{|l|}{ Verbal Learning } \\
\hline - Hopkins VLT-revised & $38.3(13.7)$ & $46.7(19.4)$ & $57.1(11.3)$ & 0.26 & .35 & .001 & .11 \\
\hline \multicolumn{8}{|l|}{ Visual Learning } \\
\hline - BVMT-revised & $45(13.8)$ & $51.2(8.4)$ & $55.2(6.9)$ & 0.17 & .24 & .07 & .53 \\
\hline
\end{tabular}

$T M-A$ trail-making test A, $C P T$ continuous performance test-identical pairs, $L N S$ letter-number span, $N A B$ neuropsychological assessment battery, $V L T$ verbal learning test, $B V M T$ brief visuospatial memory test

${ }^{\text {a }} P$ values are corrected for Bonferroni group comparisons

${ }^{\mathrm{b}}$ Missing data for 4 subjects

${ }^{\mathrm{c}}$ Missing data for 4 subjects

${ }^{\mathrm{d}}$ Missing data for 1 subject

Within patients, the $\left[\mathrm{Glu}_{\mathrm{mPFC}}\right]$-decline with age was found in EP users $(r=-.46 ; p=0.04)$ but not in EP non-users. In EP users, these results remain significant when controlling for Cpz equivalents (Fig. 4).

\section{Discussion}

To the best of our knowledge, this is the first study investigating the effect of cannabis use at neurochemical level in a representative sample of EP patients, comparing their nonusers counterpart and normative control population. Our main finding, which is in line with our principal hypothesis, is that cannabis use is significantly associated with decreased prefrontal Glu levels. Additional analyses also revealed that working memory performance was impaired in cannabis users as compared to non-users. Furthermore, our results go further and suggest that cannabis is implicated in the stronger Gludecline with age, with some relevance for the progression of the disease.
Fig. $2\left[\mathrm{Glu}_{\mathrm{mPFC}}\right]$ was lower in EP users comparing to both $\mathrm{HC}$ and EP non-users, while no differences were observed between EP non-users and controls. From left to right $\left[\mathrm{Glu}_{\mathrm{mPFC}}\right]$ in healthy controls, EP non-users and EP users, respectively. Each horizontal bar indicates the mean value of the group. $* * P=.001$; $* P=.01$

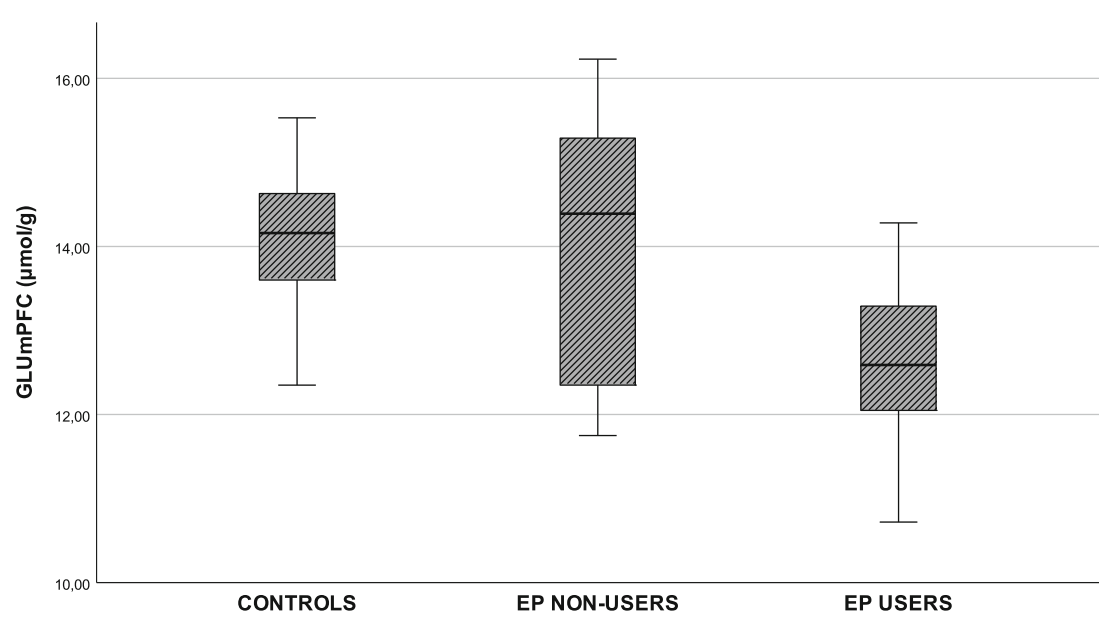



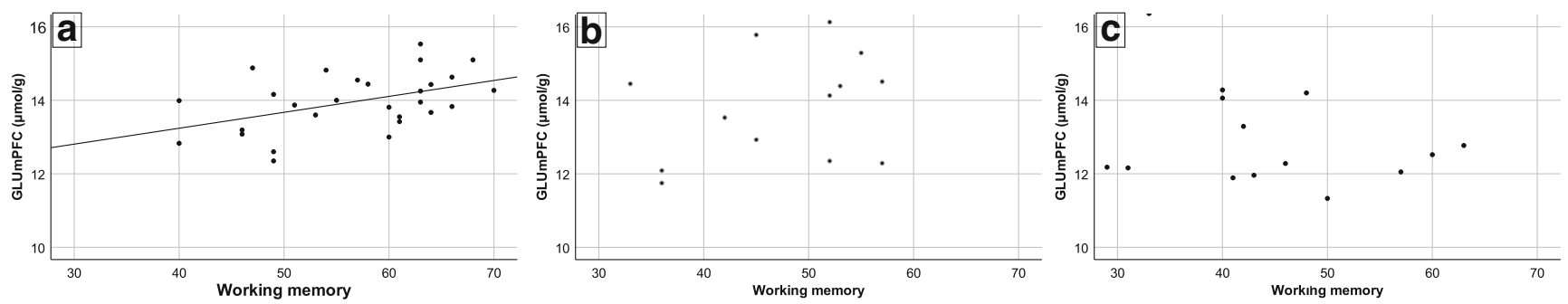

Fig. $3\left[\mathrm{GLU}_{\mathrm{mPFC}}\right]$ levels and working memory (WM) were significantly positive correlated in a healthy controls $(r=.43, p=.01)$. EP users and controls demonstrated a trend in differences in correlation coefficients of $\left[\mathrm{GLU}_{\mathrm{mPFC}}\right]$ with $\mathrm{WM},(\mathrm{z}=1.4, p=0.07)$.

\section{[Glu $\mathrm{mPFC}_{\mathrm{mP}}$ in EP cannabis-users, non-users and healthy subjects}

Our finding of reduced [Glu $\mathrm{mPFC}_{\text {] }}$ levels in EP users is consistent with the decreased Glu/Glx metabolite levels previously reported in the anterior cingulated-cortex (Muetzel et al. 2013; Prescot et al. 2013) and in basal ganglia (Chang et al. 2006; Prescot et al. 2011) of adolescent cannabis users. So far, only one study has been conducted in a cohort of early phase schizophrenia patients, reporting decreased frontal Glx levels in currently or past multi-substances users (Bernier et al. 2016). Making comparisons across studies is difficult: firstly, the outcome measure vary between studies, involving Glx metabolite measurement for two studies (Muetzel et al. 2013; Bernier et al. 2016) and Glu metabolite measurement for the others (Chang et al. 2006; Prescot et al. 2011, 2013). Secondly, the reference for quantification of Glu levels is different across studies; some studies using water (Prescot et al. 2011, 2013), while others use cerebrospinal fluid, total creatine, or phosphocreatine + creatine (Chang et al. 2006; Muetzel et al. 2013).

Discussing our findings is also complicated due to the lack of exhaustive evidence in the field. So far, only a few studies have focused on Glu-abnormalities in the frontal region before the full blown onset of the disorder (Treen et al. 2016), in EP and established schizophrenia; with some evidence that glutamatergic variations might be sensitive to illness stage (Merritt et al. 2016). It is well known, that the severity of symptoms associated with psychotic disorders typically evolves over time. Before the onset of a frank psychosis, subjects often present attenuated psychotic symptoms, usually showing a marked decline in social and occupational functioning, a clinical state referred to as Ultra High Risk (UHR) for psychotic disorder (Yung et al. 2005). Starting from this prodromal phase, progressive changes in brain volume, including the PFC, and disturbances in Glu-neurotransmission (Marsman et al. 2013) may occur. Tibbo et al. (2004) found significantly higher levels of Glu in the right medial frontal lobe of HR compared to controls, although other studies failed to replicate these findings. Purdon et al. (2008) finding the absence of differences in Glu metabolites levels between HR and HC in the same brain region, as was for Yoo et al. (2009). Concordantly, analyzing the medial-PFC in HR subjects, EP and schizophrenia patients, Natsubori et al. (2014) revealed significant decrease in Glu metabolites only in the schizophrenia group.

The decreased prefrontal Glu levels observed in EP users may be cautiously interpreted as the result of the interaction between cannabis usage and psychosis rather than the effect of illness itself, as no differences were detected between nonusers patients compared to controls. Moreover, the findings of a lack of Glu abnormalities in EP non-users may be explained in the light of those studies that did not show Glulevels decrease in the prefrontal region of EP patients, but consistently report such abnormalities in chronicity, as suggesting a role for cannabis use in driving the earlier onset of frontal Glu abnormalities. This needs however to be established in the context of a longitudinal study.
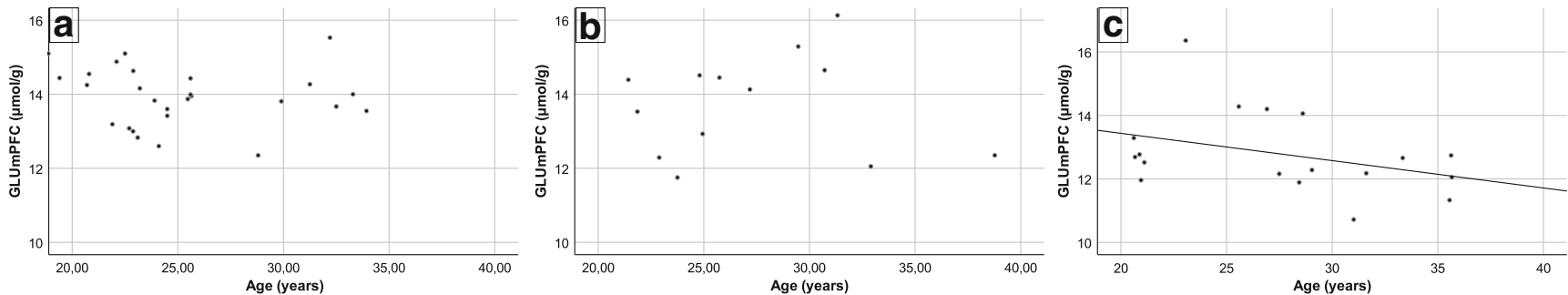

Fig. $4\left[\mathrm{GLU}_{\mathrm{mPFC}}\right]$ levels and age were: negatively correlated in $\mathbf{c}$ EP users $(r=-.46, p=.04)$, not significantly related in $\mathbf{b}$ EP non-users and a healthy controls 


\section{[Glu $\mathrm{GPFC}_{\mathrm{m}}$, age and cognition}

The decrease of $\left[\mathrm{Glu}_{\mathrm{mPFC}}\right]$ in our EP-users is intriguing and may be related to the behavioral changes observed in cannabis users. The glutamatergic system plays an important role in age-related cognitive decline and cognitive disorders, as tight regulation of Glu is essential for normal cognitive function (Rubino et al. 2015). In particular, glutamatergic transmission, that controls recurrent excitation within PFC networks, is a crucial modulator for working memory (WM) (GoldmanRakic 1995), by supporting retention of spatial information during the delay period of WM tasks (Deco and Rolls 2003). Exogenous THC exposure induces spatial WM deficits, throughout a modulation of Glu release, whose molecular underpinnings could be the establishment of less synaptic contacts and/or less efficient synaptic connections, in those brain regions like PFC that have high density of $\mathrm{CB} 1$ receptors (Rubino et al. 2009). These abnormalities may be reflected at neurochemical level by the reduction of Glu concentration seen in our EP users. Consistently with these observations, LNS (Letter Number Span), a putative executive function working memory test requiring among others attention and temporary storage of information capacities (Twamley et al. 2006), was the only neuropsychological test, albeit the limitation of the small sample size, which was impaired in EP users compared to non-users, and displayed a disrupted correlation to Glu concentration in cannabis users. Based on this background, the lack of a significant correlation between Glu levels and WM performance, observed in EP users, might probably stands for an alteration in WM signaling pathways, mediated by the reduced frontal glutamatergic tone related to cannabis use.

Counterintuitively, prior studies in EP demonstrated that subjects who used cannabis (especially those who used prior to age 16) performed better than non-using on global cognitive functioning, including WM. Moreover, these studies evidenced that the cognitive profile depends on the age at which regular cannabis use begins, and that comorbid cannabis use is associated with a superior cognitive profile only in schizophrenia-spectrum disorders but not in bipolar psychosis (Hanna et al. 2016). We failed to replicate these observations, EP users displayed poorer performances in all neuropsychological domains explored, compared both to EP non-users and HC. In addition, age at which cannabis use started was not related to cognitive performances either. Probably this could be explained by the fact that our patients were late onset users, starting cannabis consuming on average at age 17 , and heterogeneous in terms of diagnosis.

Current evidence has shown that Glu have also considerable potential as biomarker of disease progression (Brandt et al. 2016), although the implications of changes in Glu on neuronal function are still not clear (Wijtenburg et al. 2015). Given the tight metabolic relationship between glutamate and both NAA and Gln, changes in Glu levels that are not accompanied by NAA or Gln changes are surprising. NAA is an intermediate metabolite serving as a reservoir for replenishing Glu when necessary (Clark et al. 2006). Similarly, Glu and Gln are metabolically coupled, as Glu is released into the synaptic cleft and rapidly transported into astroglia where it is enzymatically converted into Gln. Subsequently, Gln is shuttled back into neurons for conversion to Glu. Our finding of a diminished glutamatergic tone and of a greater Gludecline with age in EP users, without significant related changes in NAA or Gln (Supplementary material), is only partially in line with the results of the only existing study investigating Glu-metabolites in schizophrenia patients users (Bernier et al. 2016). The authors found a reduction in Glx and mIns levels related to cannabis, alcohol, and methamphetamine use but failed to include patients without a lifetime history of substance use (non-users). Differences in both consuming behaviors (polysubstance vs cannabis use) and duration of illness between the studies may have probably accounted for these discrepancies.

\section{Strengths and limitations}

This study has a number of strengths. We have evaluated the role of cannabis in relation to brain neurochemical properties for the first time in a representative EP sample. The impact of cannabis substance used was studied independently of other substances. In addition, considering all our patients were in the early phase of psychosis, cannabis use had occurred prior to (or around) illness onset and not as a consequence of the illness. Also, patients were not exposed to long-term pharmacological treatment, making it unlikely that Glu alterations were due to antipsychotic medications. Moreover, when covarying for mean antipsychotic dosage, all our results retain their significance.

Several limitations must be noted. First of all, this study did not include a group of controls with a lifetime history of cannabis use, the real clinical-setting nature of the study might account for this. Moreover, the cross-sectional design, does not allow for a strong cause-effect interpretation of the findings. Furthermore, although other studies that have used self-report measures have also shown an association with brain structural alterations (Rigucci et al. 2016), the lack of objective measures of cannabis use in our study is an important limitation. However, there is published evidence indicating that asking patients with psychosis about their use of cannabis is, at least in some situations, more accurate than, or as reliable as, urine or blood testing which can only provide information on recent use (Freeman et al. 2014). The lack of a measure of the potency of cannabinoid compounds might nevertheless also affect the robustness of our findings; indeed, the recent changes in the composition of "street" cannabis have created a new and complex 
landscape for the understanding the neurobiological impact of cannabis products. In line with the new tendencies in Europe, Switzerland cannabis drug market has recently changed, with an increased mean THC content both in resin and herbal strains (Data from the addiction monitoring in Switzerland, 2015, http://www.suchtmonitoring.ch). Future studies evaluating the effects of cannabis use on brain structure and function should therefore include a careful assessment of cannabis potency. Differences in paradigms used in MRS, such as magnetic field strength, may influence results and need to be considered when comparing results between studies. Separating glutamate and glutamine at lower field strengths being challenging, despite promising new approaches (Zhang and Shen 2016) , and despite studies at $7 \mathrm{~T}$, having shown improved precision of glutamate measurement (Mekle et al. 2009) compared to lower field strengths, replication of these findings with an enhanced spectral sensitivity and resolution is needed.

In summary, our research which focused on prefrontal region revealed that cannabis use in the early phase of psychosis is associated with decreased Glu levels in the PFC and with a stronger Glu-decline with age, without significant changes in NAA or Gln. Our results suggest that cannabis might accelerate the progression of psychosis, which is seen at neurochemical levels with prefrontal Glu-abnormalities in EP users that normally are not found in the early stage of the illness.

Acknowledgements We would like to thank all patients and control subjects for their enduring participation. We are grateful for support from the Loterie Romande, Avina Foundation, Damm-Etienne Foundation, Pro Scientia et Arte Foundation, Alamaya Foundation and Sapienza University of Rome- - "Avvio alla Ricerca."

Funding This work was supported by the Swiss National Science Foundation (320030_122419 to P.C. and K.Q.D), National Center of Competence in Research (NCCR) "SYNAPSY (P.K and L.A - The Synaptic Bases of Mental Diseases" financed by the Swiss National Science Foundation ( ${ }^{\circ}$ 51AU40_125759). P.S.B. was supported by the Leenaards and Jeantet foundation. Magnetic resonance spectroscopy war performed in the Centre d'Imagerie BioMédicale (CIBM) of the UNIL, UNIGE, HUG, CHUV, EPFL.

\section{References}

American Psychiatric Association (1994) Diagnostic and statistical manual of mental disorders: DSM-IV. American Psychiatric Association, Washington, DC

Andreasen NC, Pressler M, Nopoulos P, Miller D, Ho B-C (2010) Antipsychotic dose equivalents and dose-years: a standardized method for comparing exposure to different drugs. Biol Psychiatry 67:255-262

Arseneault L, Cannon M, Poulton R, Murray R, Caspi A, Moffitt TE (2002) Cannabis use in adolescence and risk for adult psychosis: longitudinal prospective study. BMJ 325:1212-1213

Baumann PS, Crespi S, Marion-Veyron R, Solida A, Thonney J, Favrod J, Bonsack C, Do KQ, Conus P (2013) Treatment and early intervention in psychosis program (TIPP-Lausanne): implementation of an early intervention programme for psychosis in Switzerland. Early Interv Psychiatry 7:322-328

Bernier D, Bartha R, McAllindon D, Hanstock CC, Marchand Y, Dillen KN, Gallant M, Good KP, Tibbo PG (2016) Illness versus substance use effects on the frontal white matter in early phase schizophrenia: a 4Tesla (1)H-MRS study. Schizophr Res 175:4-11

Brandt AS, Unschuld PG, Pradhan S, Lim IA, Churchill G, Harris AD, Hua J, Barker PB, Ross CA, van Zijl PC, Edden RA, Margolis RL (2016) Age-related changes in anterior cingulate cortex glutamate in schizophrenia: a (1)H MRS Study at 7 Tesla. Schizophr Res 172: 101-105

Chang L, Cloak C, Yakupov R, Ernst T (2006) Combined and independent effects of chronic marijuana use and HIV on brain metabolites. J Neuro Pharmacol 1:65-76

Clark JF, Doepke A, Filosa JA, Wardle RL, Lu A, Meeker TJ, PyneGeithman GJ (2006) N-acetylaspartate as a reservoir for glutamate. Med Hypotheses 67:506-512

Colizzi M, McGuire P, Pertwee RG, Bhattacharyya S (2016) Effect of cannabis on glutamate signalling in the brain: a systematic review of human and animal evidence. Neurosci Biobehav Rev 64:359-381

Deco G, Rolls ET (2003) Attention and working memory: a dynamical model of neuronal activity in the prefrontal cortex. Eur J Neurosci 18:2374-2390

Fontes MA, Bolla KI, Cunha PJ, Almeida PP, Jungerman F, Laranjeira RR, Bressan RA, Lacerda AL (2011) Cannabis use before age 15 and subsequent executive functioning. Br J Psychiatry 198:442-447

Freeman TP, Morgan CJ, Hindocha C, Schafer G, Das RK, Curran HV (2014) Just say 'know': how do cannabinoid concentrations influence users' estimates of cannabis potency and the amount they roll in joints? Addiction 109:1686-1694

Goldman-Rakic PS (1995) Cellular basis of working memory. Neuron 14:477-485

Gruetter R (1993) Automatic, localized in vivo adjustment of all first- and second-order shim coils. Magn Reson Med 29:804-811

Gruetter R, Tkac I (2000) Field mapping without reference scan using asymmetric echo-planar techniques. Magn Reson Med 43:319-323

Hanna RC, Shalvoy A, Cullum CM, Ivleva EI, Keshavan M, Pearlson G, Hill SK, Sweeney JA, Tamminga CA, Ghose S (2016) Cognitive function in individuals with psychosis: moderation by adolescent cannabis use. Schizophr Bull 42:1496-1503

Howes O, McCutcheon R, Stone J (2015) Glutamate and dopamine inschizophrenia: an update for the 21 st century. J Psychopharmacol 29:97-115

Liemburg E, Sibeijn-Kuiper A, Bais L, Pijnenborg G, Knegtering H, van der Velde J, Opmeer E, de Vos A, Dlabac-De Lange J, Wunderink L, Aleman A (2016) Prefrontal NAA and Glx levels in different stages of psychotic disorders: a 3T 1H-MRS study. Sci Rep 6(1):21873

Lorenzetti V, Solowij N, Yücel M (2015) The role of cannabinoids in neuroanatomic alterations in cannabis users. Biol Psychiatry 79:1731

Marsman A, van den Heuvel MP, Klomp DW, Kahn RS, Luijten PR, Hulshoff Pol HE (2013) Glutamate in schizophrenia: a focused review and meta-analysis of ${ }^{1} \mathrm{H}-\mathrm{MRS}$ studies. Schizophr Bull 39:120 129

Mato S, Robbe D, Puente N, Grandes P, Manzoni OJ (2005) Presynaptichomeostatic plasticity rescues long-term depression after chronic Delta9-tetrahydrocannabinol exposure. J Neurosci 25: 11619-11627

Mekle R, Mlynarik V, Gambarota G, Hergt M, Krueger G, Gruetter R (2009) MR spectroscopy of the human brain with enhanced signal intensity at ultrashort echo times on a clinical platform at 3T and 7T. Magn Reson Med 61:1279-1285

Merritt K, Egerton A, Kempton MJ, Taylor MJ, McGuire PK (2016) Nature of glutamate alterations in schizophrenia: a meta-analysis of proton magnetic resonance spectroscopy studies. JAMA Psychiatry 73:665-674 
Mlynarik V, Gambarota G, Frenkel H, Gruetter R (2006) Localized shortecho-time proton MR spectroscopy with full signal-intensity acquisition. Magn Reson Med 56:965-970

Muetzel RL, Marjanska M, Collins PF, Becker MP, Valabregue R, Auerbach EJ, Lim KO, Luciana M (2013) In vivo H magnetic resonance spectroscopy in young-adult daily marijuana users. NeuroImage Clin 2:581-589

Mugler JP 3rd, Brookeman JR (1990) Three-dimensional magnetizationprepared rapid gradient-echo imaging (3D MP RAGE). Magn Reson Med 15:152-157

Natsubori T, Inoue H, Abe O, Takano Y, Iwashiro N, Aoki Y, Koike S, Yahata N, Katsura M, Gonoi W, Sasaki H, Takao H, Kasai K, Yamasue H (2014) Reduced frontal glutamate + glutamine and Nacetylaspartate levels in patients with chronic schizophrenia but not in those at clinical high risk for psychosis or with first-episode schizophrenia. Schizophr Bull 40:1128-1139

Nuechterlein KH, Green MF, Kern RS, Baade LE, Barch DM, Cohen JD, Essock S, Fenton WS, Frese FJ 3rd, Gold JM, Goldberg T, Heaton RK, Keefe RS, Kraemer H, Mesholam-Gately R, Seidman LJ, Stover E, Weinberger DR, Young AS, Zalcman S et al (2008) The MATRICS consensus cognitive battery, Part 1: test selection, reliability, and validity. Am J Psychiatry 165:203-213

Pistis M, Porcu G, Melis M, Diana M, Gessa GL (2001) Effects of cannabinoids on prefrontal neuronal responses to ventral tegmental area stimulation. Eur J Neurosci 14:96-102

Preisig M, Fenton BT, Matthey ML, Berney A, Ferrero F (1999) Diagnostic interview for genetic studies (DIGS): inter-rater and test-retest reliability of the French version. Eur Arch Psychiatry Clin Neurosci 249:174-179

Prescot AP, Locatelli AE, Renshaw PF, Yurgelun-Todd DA (2011) Neurochemical alterations in adolescent chronic marijuana smokers: a proton MRS study. NeuroImage 57:69-75

Prescot AP, Renshaw PF, Yurgelun-Todd DA (2013) Amino butyric acid andglutamate abnormalities in adolescent chronic marijuana smokers. DrugAlcohol Depend 129:232-239

Purdon SE, Valiakalayil A, Hanstock CC, Seres P, Tibbo P (2008) Elevated $3 \mathrm{~T}$ proton MRS glutamate levels associated with poor Continuous Performance Test (CPT-0X) scores and genetic risk for schizophrenia. Schizophr Res 99:218-224

Rigucci S, Marques TR, Di Forti M, Taylor H, Dell'Acqua F, Mondelli V, Bonaccorso S, Simmons A, David AS, Girardi P, Pariante CM, Murray RM, Dazzan P (2016) Effect of high-potency cannabis on corpus callosum microstructure. Psychol Med 46:841-854

Rubino T, Realini N, Braida D, Alberio T, Capurro V, Viganò D, Guidali C, Sala M, Fasano M, Parolaro D (2009) The depressive phenotype induced in adult female rats by adolescent exposure to THC is associated with cognitive impairment and altered neuroplasticity in the prefrontal cortex. Neurotox Res 15:291-302
Rubino T, Prini P, Piscitelli F, Zamberletti E, Trusel M, Melis M, Sagheddu C, Ligresti A, Tonini R, Di Marzo V, Parolaro D (2015) Adolescent exposure to THC in female rats disrupts developmental changes in the prefrontal cortex. Neurobiol Dis 73:60-69

Tibbo P, Hanstock C, Valiakalayil A, Allen P (2004) 3-T proton MRS investigation of glutamate and glutamine in adolescents at high genetic risk for schizophrenia. Am J Psychiatry 161:1116-1118

Tkac I, Starcuk Z, Choi IY, Gruetter R (1999) In vivo 1H NMR spectroscopy of rat brain at $1 \mathrm{~ms}$ echo time. Magn Reson Med 41:649-656

Tkac I, Andersen P, Adriany G, Merkle H, Ugurbil K, Gruetter R (2001) In vivo $1 \mathrm{H}$ NMR spectroscopy of the human brain at $7 \mathrm{~T}$. Magn Reson Med 46:451-456

Treen D, Batlle S, Mollà L, Forcadell E, Chamorro J, Bulbena A, Perez V (2016) Are there glutamate abnormalities in subjects at high risk mental state for psychosis? A review of the evidence. Schizophr 171:166-175

Twamley EW, Palmer BW, Jeste DV, Taylor MJ, Heaton RK (2006) Transient and executive function working memory in schizophrenia. Schizophr Res 87:185-190

Wijtenburg SA, Yang S, Fischer BA, Rowland LM (2015) In vivo assessment of neurotransmitters and modulators with magnetic resonance spectroscopy: application to schizophrenia. Neurosci Biobehav Rev 51:276-295

Xin L, Mekle R, Fournier M, Baumann PS, Ferrari C, Alameda L, Jenni R, Lu H, Schaller B, Cuenod M, Conus P, Gruetter R, Do KQ (2016) Genetic polymorphism associated prefrontal glutathione and its coupling with brain glutamate and peripheral redox status in early psychosis. Schizophr Bull 42:1185-1196

Yoo SY, Yeon S, Choi C, Kang DH, Lee JM, Shin NY, Jung WH, Choi JS, Jang DP, Kwon JS (2009) Proton magnetic resonance spectroscopy in subjects with high genetic risk of schizophrenia: investigation of anterior cingulate, dorsolateral prefrontal cortex and thalamus. Schizophr Res 111:86-93

Yung AR, Yuen HP, McGorry PD, Phillips LJ, Kelly D, Dell'Olio M, Francey SM, Cosgrave EM, Killackey E, Stanford C, Godfrey K, Buckby J (2005) Mapping the onset of psychosis: the comprehensive assessment of at-risk mental states. Aust N Z J Psychiatry 39: 964-971

Zamberletti E, Beggiato S, Steardo L Jr, Prini P, Antonelli T, Ferraro L, Rubino T, Parolaro D (2014) Alterations of prefrontal cortex GABAergic transmissionin the complex psychotic-like phenotype induced by adolescentdelta-9-tetrahydrocannabinol exposure in rats. Neurobiol Dis 63:35-47

Zhang Y, Shen J (2016) Simultaneous quantification of glutamate and glutamine by J-modulated spectroscopy at 3 Tesla. Magn Reson Med 76:725-732 